\title{
Somatopsychic Phenotype of the Bronchial Asthma: Diagnostics and Treatment
}

\author{
Tetyana Konstantynovych", Anna Demchuk, Alina Dovgan, Yuriy Mostovoy \\ Propedeutic Department to internal medicine, National Pirogov Memorial Medical University, Vinnytsya, Vinnytsia, Ukraine
}

Email address:

tvkonstantinovich@yahoo.com (T. Konstantynovych)

${ }^{*}$ Corresponding author

\section{To cite this article:}

Tetyana Konstantynovych, Anna Demchuk, Alina Dovgan, Yuriy Mostovoy. Somatopsychic Phenotype of the Bronchial Asthma: Diagnostics and Treatment. American Journal of Internal Medicine. Special Issue: New Approaches to Manage Difficult-to-Control, Severe Asthma. Vol. 8, No. 2, 2020, pp. 66-72. doi: 10.11648/j.ajim.20200802.14

Received: February 7, 2020; Accepted: February 27, 2020; Published: March 10, 2020

\begin{abstract}
The investigation is devoted to the problem of improvement of diagnostics and treatment of the somatopsychic phenotype (SPPh) at patients with bronchial asthma (BA). On the basis of complex clinical, instrumental, psychological inspection, investigation of the parameters of life quality by MOS SF-36 questionnaire of 207 BA-patients certain prevalence of the combination of concomitant psycho-emotional disorders in $39.1 \%$ of BA patients, such as high levels of anxiety, neurotism, clinically significant depressive symptoms and somatopsychological accentuations (pedantry, emotionality, anxiety, excitability, cyclothymia and dysthymia). It is established, that BA patients have the somatopsychic phenotype of the course of the disease. We described the features of clinical course of BA, lung functional parameters and indexes of quality of life at patients with the pathologically changed somatopsychological descriptions. It is associated with an increase in the clinical severity of the disease, a decrease in the lung function parameters, low quality of life indexes and absence of asthma control. Assignment of psychotropic drugs (fabomotisol dihydrochloride (afobazole) or piracetam/thiotriazoline) in the complex treatment leads to stabilization of the clinical and psychological state of patients, increasing the level of control of asthma. The clinical and economic effectiveness of using of the combined modes of therapy with including of preparations of psychotropic action on a base treatment was proved.
\end{abstract}

Keywords: Bronchial Asthma, Somatopsychic Phenotype, Life Quality, Diagnostics, Treatment

\section{Introduction}

Bronchial asthma (BA) continues to be one of the major health problems in the world. According to official statistics, the prevalence of asthma in the world is from 1 to $18 \%$, it is the cause of about 346000 deaths worldwide $[1,2]$. Therefore, it is important to look for reasons that cause the progression of asthma and reduce the control of the disease.

\section{Theoretical Background}

Increasingly in recent years, it is said that asthma is not the total diagnosis, but a clinical syndrome, heterogeneous in its manifestations, pathophysiological mechanisms, features of inflammation [3]. The heterogeneous nature of asthma is emphasized in the GINA guidance in the new definition of the disease $[1,2,4]$, and the concept of asthma phenotype
[5], which should be taken into account in the diagnosis and choice of treatment regimen, is increasingly being introduced into clinical practice.

Recent studies have confirmed a clear tendency for an increase in the proportion of BA patients with comorbid diseases, including arterial hypertension, coronary heart disease, diabetes, and depression [3, 6-8]. It is recognized that neurological and mental factors are important components of the pathogenetic complex that determines the course and prognosis of asthma [9-11].

A number of international documents on the problems of asthma are actively discussing the problem of further study of psychological features in their organic relationship with the course of the disease, since in many cases it is the emotional and mental factors that explain the decline in the level of compliance and asthma control [12-15]. Mental disorders, especially depressive and anxious conditions, 
prevail in persons with asthma. They are associated with worsening asthma control, decreased adherence to therapy, and poor quality of life $[1,2,4,16,17]$.

There are divergent views on the problem of treatment of patients with asthma in the presence of psychological disorders [6, 15-17]. The number of qualitative studies on the evaluation of pharmacological and non-pharmacological methods of treatment of disorders of the emotional sphere in patients with asthma is small, and the results obtained in these studies are inconsistent [18]. Drug and cognitivebehavioral therapy are believed to have some potential for patients with asthma. However, the evidence currently available is limited by a small amount of research and methodological shortcomings [1, 2]. All of the above have made the research relevant.

The aim of the study: on the basis of establishing the frequency and features of the course of somatopsychic phenotype (SPPh) in patients with asthma, improve the diagnosis and increase the effectiveness of treatment of this disease through medical correction of fixed disorders of the emotional and mental sphere.

\section{Material and Experiments}

The basis of the research was the observation of 207 inpatients with asthma and were subject to further supervision at the outpatient stage. Among them, there were $80(38.6 \%)$ men and $127(61.4 \%)$ women, the average age $(42.1+0.9)$ years old. The severity of BA and the level of control were determined according to national and international GINA approaches [1, 2]. A mild persistent course was diagnosed in $26(12.5 \%)$ ones, moderate - 95 $(45.9 \%)$ ones, severe - $86(41.5 \%)$ ones.

The average duration of asthma was $(12.5+8.6)$ years. Concomitant diseases in patients with BA were in the stage of complete clinical and functional compensation for at least 0.5 year before the examination. The control group consisted of 82 practically healthy individuals $(27$ (32.9\%) men, 55 $(67.1 \%)$ women), who were representative to the main group by age, social-economic status, and prevalence of comorbidities.

Patients with BA were examined on a specially designed original card with information of sex, age, anthropometric data, social-economic status, frequency and severity of symptoms of asthma, indicators of physical activity, laboratory and instrumental and consultative examinations at the inpatient and outpatient stage treatment. The control level was determined using the AST test [19]. At the time of the examination, patients were treated permanently and had an uncontrolled disease course due to exacerbation. The degree of ventilation failure was determined using a MasterScopePC computer spirograph (Erich Jaeger, Germany) according to European guidelines [20].

At the 1st step of the study, all the respondents were diagnosed with somatopsychic phenotype (SPPh), based on a clinical-experimental approach, which included: orientation diagnostic interview, determination of types of personality accentuation by K. Leonhard - H. Shmishek method [21], diagnosis of SPPh establishments and gravity. The level of state anxiety (SAL) and trait anxiety (TAL) was determined using C. D. Spielberger-Y. L Hanin scale (State-Trait Anxiety Inventory) (2002). The level of neurotization (NL) was determined as a percentage (\%) by LI. Wasserman (2002). Diagnosis of depressive states was performed using The Zung self-rating depression scale (1971) in adaptation by TI Balashova [22].

At the second stage of the study, a sample of 78 outpatients (mild BA - 8, moderate asthma - 40, severe asthma - 30) was formed to determine the clinical and functional efficacy of treatment of patients with asthma, along with traditional basic treatment, using psychotropic drugs. activity. When choosing psychotropic medicines, they were guided by the criteria of their compliance with the specificity of patients with asthma, in fact: use of psychotropic medication in monotherapy; preference was given to the number with the multidisciplinary effects of pharmacological effects, their satisfactory interaction with basic drugs, minimal behavioral toxicity and the need for titration in the absence of effects of bronchoconstriction, inhibition of the respiratory center and processes of physiological drainage of the bronchial tree, economic accessibility. Patients were divided into three clinical groups by random sampling. Informed patient consent was obtained from the studies.

The first mode of treatment involved the use of only drugs of basic treatment of BA according to a step-approach to severity $[1,2]$ - inhaled GCS (budesonide or beclomethasone dipropionate) in accordance with the severity of the disease daily dose, combined with long-acting $\beta 2$-agonist formoterol or salmeterol).

In the second mode, patients were randomized into 2 subgroups: II-A and II-B.

The II-A subgroup consisted of patients who, in addition to baseline therapy, received medication with anxiolytic activity of fabomotisol dihydrochloride (afobazole) at a dose of 0.01 gr three times a day.

Fabomotisol dihydrochloride (afobazole) is a selective non-benzodiazepine anxiolytic. Acting on sigma-1 receptors in nerve cells of the brain, it stabilizes GammaAminoButyric Acid (GABA)/benzodiazepine receptors and restores their sensitivity to endogenous inhibitory mediators. It also increases the bioenergetic potential of neurons and has a neuroprotective effect: restores and protects nerve cells.

The action of the drug is implemented mainly in the form of a combination of anxiolytic (anti-anxiety) and mild stimulating (activating) effects. The drug reduces or eliminates feelings of anxiety, irritability, tension, depressive mood, somatic manifestations of anxiety, autonomic disorders, cognitive impairment, including arising from stress disorder adaptation. The drug is indicated for individuals with predominantly asthenic personality traits in the form of anxious suspiciousness, uncertainty, increased vulnerability and emotional lability, a tendency to emotional-stress reactions. The drug is one of the few that are approved for use in anxiety and neurotic conditions, adaptation disorders, 
and in patients with bronchial asthma [23].

The II-B subgroup consisted of patients receiving the drug, which is a fixed combination of piracetam / thiotriazoline, belongs to the group of psychostimulants and nootropic agents, at a dose of $0.4 / 0.1 \mathrm{~g}$ three times a day.

The drug belongs to the group of cerebroactive drugs, has anti-ischemic, antioxidant, membrane-stabilizing and nootropic properties. The drug improves the integrative and cognitive activity of the brain, increases the efficiency of the learning process, helps to eliminate the symptoms of amnesia, and increases the indicators of short-term and longterm memory [24]. The pharmacological effect of the drug is due to the potentiating effect of thiotriazolin and piracetam. The drug is able to accelerate the oxidation of glucose in the reactions of aerobic and anaerobic oxidation, normalize bioenergy processes, increase adenosine-three-phosphate (ATPh) level, stabilize metabolism in brain tissues. The drug inhibits the formation of reactive oxygen species, reactivates the antioxidant system of enzymes, especially superoxide dismutase, inhibits free radical processes in the brain tissue during ischemia, improves the rheological properties of blood by activating the fibrinolytic system, stabilizes and reduces, respectively, areas of necrosis and ischemia.

The combined use of piracetam and thiotriazolin is an innovative direction in the treatment of initial brain lesions associated with prolonged exposure to damaging factors, including hypoxia, metabolic disorders of various origins, vascular disorders, which is especially true for chronic lung diseases, including obstructive, which results in the development of chronic cerebral deficiency and the development of hypoxic dysmetabolic encephalopathy [25].

The average treatment period was $(30+2)$ days. Before and after treatment, clinical symptoms of asthma, lung function parameters, ACT-test results, dynamics of psycho-emotional indicators and quality of life (QoL) using the MOS SF-36 questionnaire were evaluated [26].

Statistical processing of the results was performed on a personal computer using the SPSS 12.0 statistical software package for Windows (Grand Pack License Serial Number 9593869). The results of the comparisons with a probability of error $\mathrm{p}<0.05$ were considered plausible [27].

\section{Results of the Study and Their Discussion}

The mental state of patients with asthma differed with probable shifts for the worse in comparison with the control group in both prevalence and severity, which testifies to the secondaryness of concomitant $\mathrm{SPPh}$ and their somatogenic conditionality. The prevalence of concomitant disorders of the psycho-emotional sphere is determined according to the degree of their expressiveness (Figure 1).

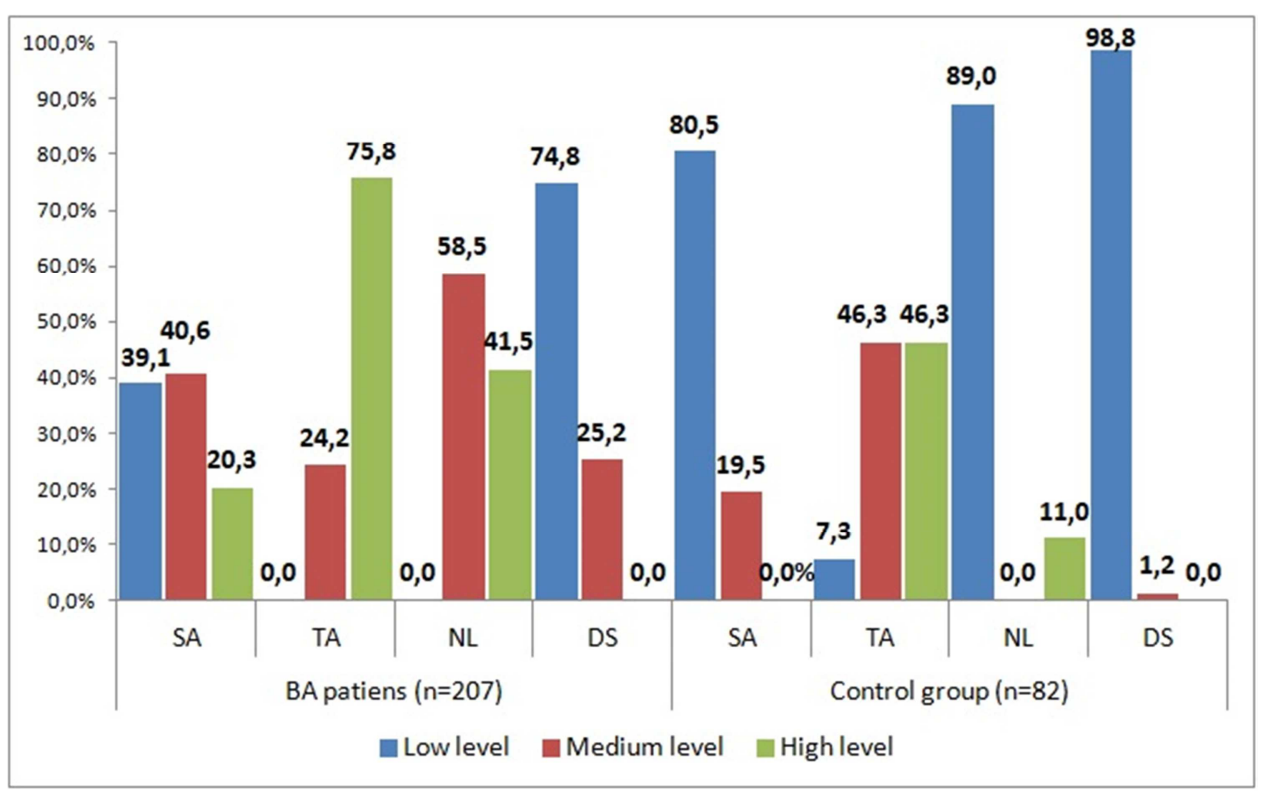

Figure 1. Structure and prevalence (\%) of somatopsychic phenotype in patients with asthma and in control subjects (SA-state anxiety, TA- trate anxiety, NL neurotization level, DS- depression states`level).

High neurotic level (NL) was determined in $86(41.5 \%)$ patients vs. $9(11.0 \%)$ in the control group $(\mathrm{p}<0.001)$, high state anxiety level (SAL) - in $42(20.3 \%)$ patients, while in control subjects it was not observed at all in the group (p $<0.001)$, high trate anxiety level (TAL) - in $157(75.8 \%)$ patients with asthma vs. $38(46.3 \%)$ among the control group $(\mathrm{p}<0.001)$, depressive states (DS) were observed in a total of $52(25.2 \%)$ patients with BA and manifested with mild depression in $44(21.3 \%)$, subdepressive condition - in 8 (3.9\%), while only 1 among the control group were diagnosed $(1.2 \%)$ case of subdepressive condition $(\mathrm{p}<0.001)$. In $81(39.1 \pm 0.03) \%$ patients during the development of asthma a SPPh of the course of asthma was formed, which was higher than the prevalence among the control group - 5 cases $((6.1+0.037) \%, \mathrm{p}<0.001)$ (Figure 2). 


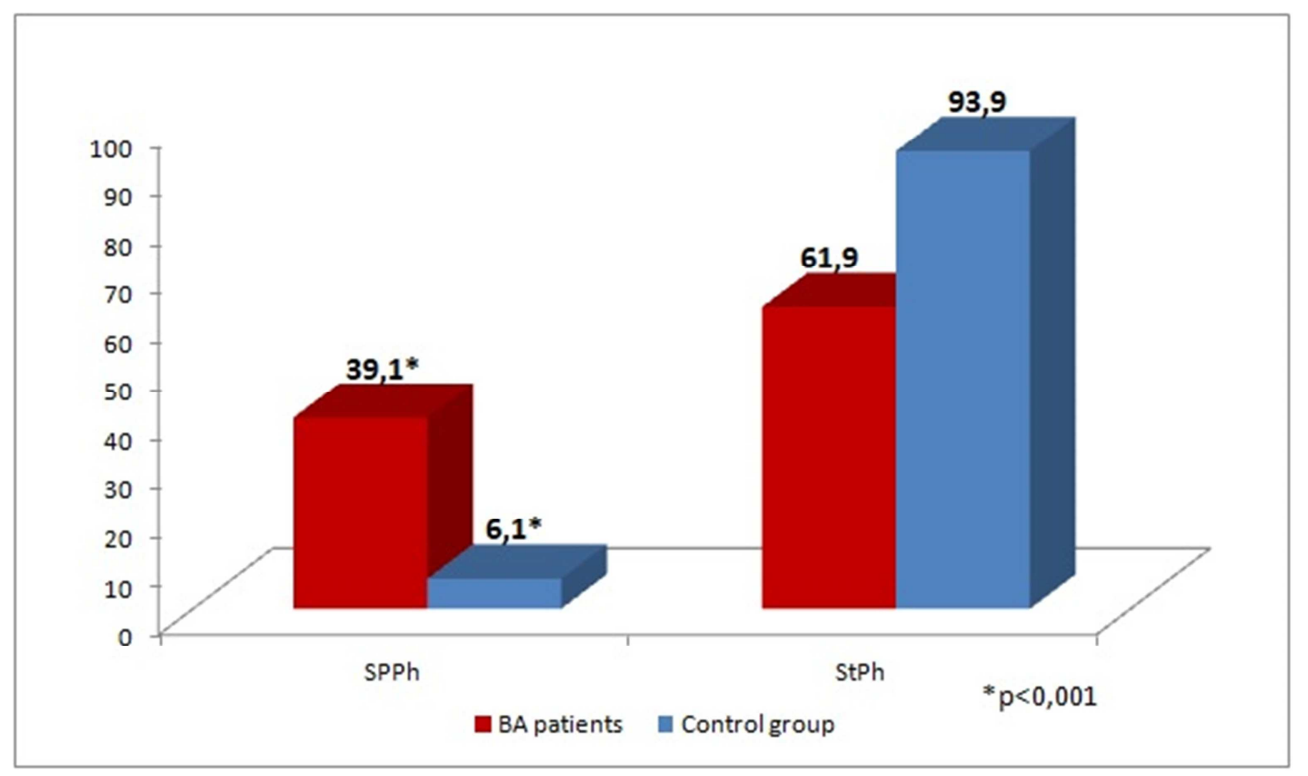

Figure 2. Prevalence (\%) of somatopsychic phenotype in patients with asthma and in control subjects.

Gender and age characteristics of SPPh of the course of asthma have been established. In women, a high level of SAL was defined in $37(29.1 \%)$, TAL - $103(81.1 \%)$, DS - 40 $(31.5 \%)$ in the form of mild depression - $32(25.2 \%)$ and subdepressive condition - $8(6.3 \%)$, NL - in $63(49.0 \%)$. Men were found to have a lower prevalence of the SPPh of BA: high SAL was determined in $5(6.3 \%),(\mathrm{p}<0.001)$, TAL - 54 $(67.5 \%),(p=0.026)$, mild-level DS (12 (15.0\%), $(\mathrm{p}=0.010)$, NL - $23(28.8 \%),(p=0.003)$. The prevalence of the SPPh of asthma was undulating on all diagnostic scales, with minimal development at young age up to 25 years, with maximum reaching at the age of 50-60 years and subsequent decrease in patients of older age groups.

As the BA progresses, the prevalence and severity of individual disorders of the psycho-emotional sphere have increased. High SAL in patients with severe disease was observed in 23.3 vs. $13.6 \%$ of patients with mild asthma $(\mathrm{p}=0.030)$, TAL -84.9 vs. $50.0 \%,(\mathrm{p}<0.001), \mathrm{NL}-46,5$ vs. $13.6 \%,(p=0.007)$, DS $-29.1 \%$ in the absence of signs of depression in mild asthma, $(\mathrm{p}=0.034)$. The presence of severe persistent BA in patients was accompanied by a combination of three syndromes with critical severity levels of $44.2 \%$ vs. $22.7 \%$ for mild persistent $\mathrm{BA}(\mathrm{p}=0.03)$.

Patients with BA differed from the group of healthy subjects with higher values of accentuation and were considered to be accentuated on the scales of pedantry, emotionality, anxiety, excitability, cyclothymia and dysthymia. Women were more stressed than men on the scale of emotion and exaltation. The study of the associative relationships of the combination of accentuation traits among patients with BA allowed us to determine the combination of traits most commonly found among patients with asthma, and was expressed by the combination of traits of pedantry, emotion, cyclothymia and dysthymia at $38(18.4+0.034) \%$.

The presence of this personality profile correlated positively with the severity of asthma $(r=0.198, p=0.004)$, which indicates that there is an impact of severe disease on the formation of personality structure. There was a gradual increase in the prevalence profile of accentuation as the severity of asthma increased - from $1(2.6 \%)$ cases for mild, to $16(42.1 \%)$ - for moderate and $21(55.3 \%)$ for severe BA.

We have analyzed the indicators of the course of asthma in groups of patients in accordance with the severity of the disease, on the one hand, and depending on the characteristics of mental status, on the other. At the same time, within the groups of patients with equal severity were singled out persons with $\mathrm{SPPh}$ course of BA and patients with clinically insignificant psycho-emotional symptoms.

In patients with mild uncontrolled BA, no significant differences were found in the frequency and severity of BA symptoms ( $>0.05)$, although the absolute values of the parameters that determine the nature of the course were more pronounced in the presence of SPPh. Patients in this group were characterized by the lowest duration of asthma (damaging factors had minimal impact on patients) and young age (which did not imply the development of physiological changes in the brain).

In the moderate, uncontrolled course of SPPh BA revealed positive correlations between the level of comorbid psychic disorders, the frequency and severity of BA symptoms. There was an increase in the daily frequency of BA-attacks $(7.2+0.8)$ versus $(5.1+0.4)$ times, $(r=0.242, p=0.018)$, the predominance of the frequency of night awakenings - $(3.5+0$, 4) versus $(2.1+0.3)$ times, $(r=0.286, p=0.005)$, the use of $\beta 2$ agonists on demand $(\mathrm{r}=0.210, \mathrm{p}=0.041)$, the average frequency of which was $(12,1+1,6)$ times/day versus $(8,7+0,7)$ times/day and the severity of cough $(\mathrm{r}=0,248$, $\mathrm{p}=0,015)$, the severity of which was estimated in $(2,4+0,1)$ against $(1.6+0.1)$ points in the comparison group. Women tended to be more severe, compared to men with the course of BA ( $\mathrm{p}<0.10)$, were characterized by likely abuse of "fast" $\beta 2$-agonists, the use of which was 2.4 times higher than that 
in men $(\mathrm{p}=0.018)$.

An analysis of the clinical symptoms of SPPh of BA, conducted in a group of patients with severe uncontrolled course, did not reveal any probable differences, except for the severity of cough, the average of which was weakly positively correlated with emotional dysfunction $(\mathrm{r}=0.221$, $\mathrm{p}=0.041$ ). From our point of view, this may indicate that in conditions of severe asthma, the negative impact of concomitant SPPh is counteracted by the constantly acting damaging factors, which include progressive hypoxia, oxidative stress, circulatory and lung disorders, and more. The result of this process is the remodeling of the respiratory system and the gradual erasure of clinical differences as the severity of the disease increases.

The correlation between the lung function values and the age of the patients, the duration and severity of BA were found. In addition to the severity of the course, the SPPh of BA has been found to have a negative effect on lung function. The most sensitive was FEF25-75, which was probably lower than the changed emotional and mental state and was $(43.7+3.9) \%$ of the corresponding versus $(53.7+3.1) \%,(p=0.039)$, in while the values of other lung function indicators did not show a significant difference between patients.

We tested the modes that involved correction of pathologically altered somatopsychic characteristics of patients with BA on the background of basic therapy. The study showed that the inclusion of psychotropic drugs in the complex treatment of patients with asthma potentiated the effects of basic therapy due to the probably faster dynamics of somatic, functional and emotional and mental characteristics of patients, improved quality of life, helped to increase the level of control over asthma during the first month (Figure 3).

The mode that prescribed the use of fabomotisol dihydrochloride (aphobazole) and piracetam/thiothiazolin (PT) were better in the background of baseline drugs $(58.6 \%$ and $42.9 \%$, respectively, compared to $32.1 \%$ compared to the traditional treatment regimen, $\mathrm{p}<0.05$ ) clinical and functional efficacy to achieve satisfactory control over a 30-day course of treatment (Figures 3, 4).

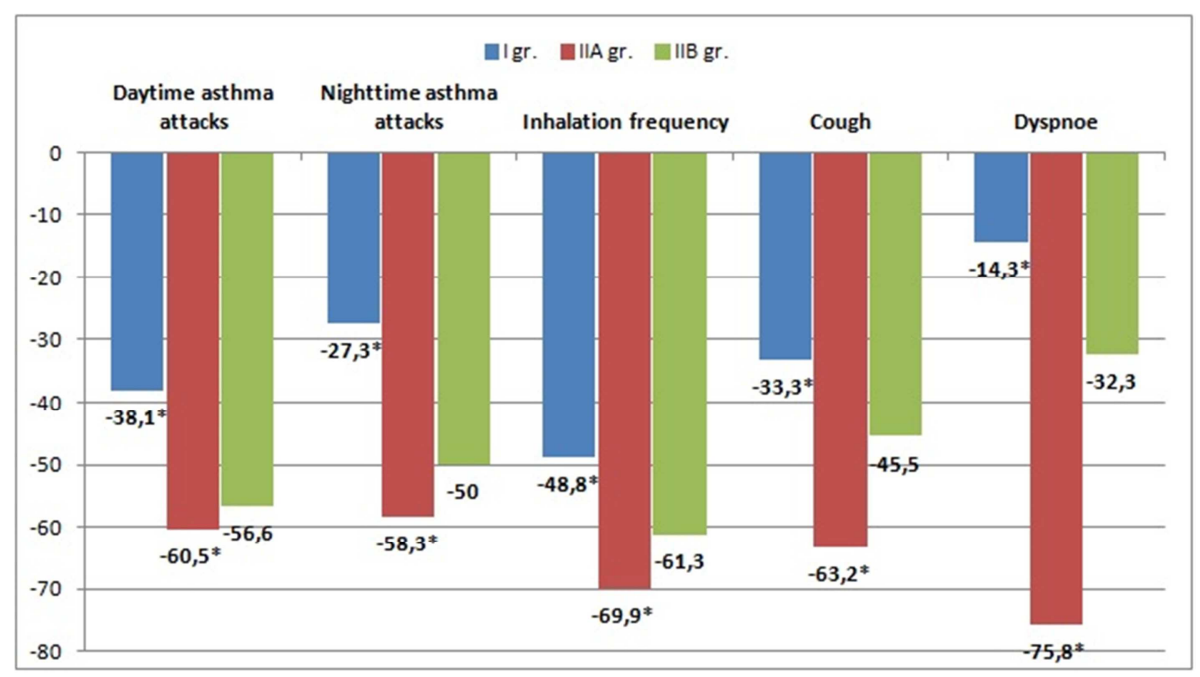

Figure 3. Dynamics of clinical symptoms of asthma in different modes of therapy $\left(*^{*}<0.05\right)$.

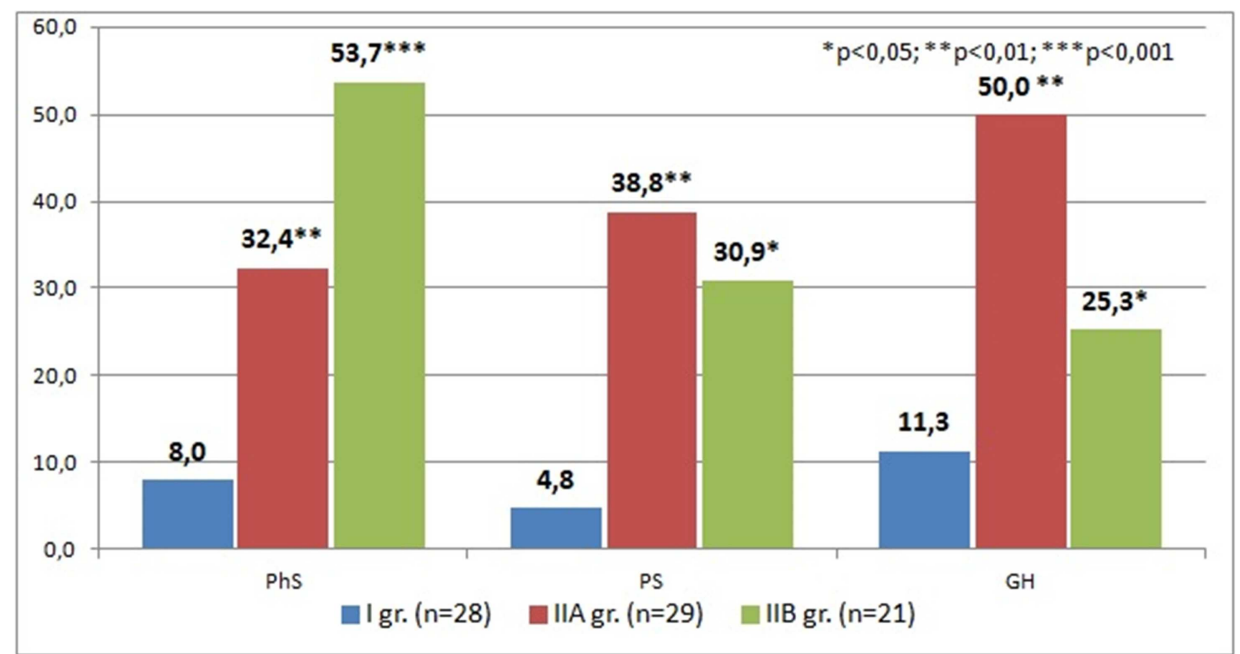

Figure 4. Increase of quality of life indicators of patients with asthma under different modes of therapy (PhS-physical status, PS-psycilogical status, GHgeneral health). 
For patients with moderate BA, the use of fabomotisol dihydrochloride (afobazole) has proven to be more clinically effective than the use of piracetam/thiothiazolin (PT). Multiple effects of psychotropic drugs have been established for patients with severe BA. The inclusion of fabomotisol dihydrochloride (aphobazole) shows a better effect on the dynamics of clinical, functional and somatopsychic characteristics of patients, probably due to its anxiolytic and anti-asthenic effects, while the inclusion of pyracetam/tiotriazolin in explain the mobilizing influence of this nootropic on all links of patients' physical, mental and social activity (Figures 3, 4).

\section{Conclusions}

1. On the background of the course of asthma in $(39.1+0.03) \%$ of cases concomitant somatopsychic dysfunction develops, which is represented by high state anxiety level $-(20.3+0.05) \%$, high trate anxiety level - $(75.8+0.03) \%$, high neurotisation level $(41,5+0,03) \%$, mild depressive states - $(25,2+0,04) \%$, which indicates the formation of somatopsychic phenotype of the of the disease.

2. Patients of the older age groups $(\mathrm{r}=0.150, \mathrm{p}=0.031)$, female $(r=0.230, p=0.001)$, with a severe course of BA $(\mathrm{r}=0.184, \mathrm{p}=0.008)$ are prone to develop somatopsychic phenotype of BA.

3. The persistent course of asthma is accompanied by the formation of a specific personality profile in $(18.4+0.03) \%$ of patients and is manifested by a combination of traits of pedantry, emotionality, cyclothymia and dysthymia; the progression of asthma is associated with an increase in features of emotionality $(\mathrm{r}=0,413, \mathrm{p}<0,001)$ and dysthymia $(\mathrm{r}=0,240, \mathrm{p}=0,026)$ and a decrease in accentuation on a scale of excitability $(\mathrm{r}=-0,240, \mathrm{p}=0,032)$, which indicates a change in mental background.

4. The course of asthma in the somatopsychic phenotype is characterized by increase in the daily frequency of attacks $(p=0.018)$, nocturnal symptoms $(p=0.005)$, the use of short-acting $\quad \beta 2$-agonists $\quad(p=0.041)$, intensification of BA symptoms in the interannual period $(p=0.015)$, worse external respiration compared with patients with a psychologically stable BA phenotype.

5. The inclusion of psychotropic drugs in the treatment of patients with asthma potentiates the effects of basic therapy due to the probably faster dynamics of somatic, functional and emotional-mental characteristics of patients, quality of life indicators, increases the level of asthma control within 30 days of treatment.

6. The obtained results have scientific and practical value and are recommended for use in practical health care in order to optimize and improve the effectiveness of the treatment of patients with asthma in the presence of a somatopsychic phenotype.

\section{References}

[1] Global Initiative for Asthma. Global Strategy for Asthma Management and Prevention. (GINA 2019) online appendix. Electronic resource: Available from: http://ginasthma.org/2019-gina-report-global-strategy-forasthma-management-and-prevention.

[2] Global Initiative for Asthma. Global Strategy for Asthma Management and Prevention. (GINA 2018) online appendix Electronic resource: Available from: http://ginasthma.org/2.2018-online-appendix-global-strategyfor-asthma-management-and-prevention.

[3] Boulet P. Influence of comorbid conditions on asthma. Eur Respir Journ, 2009; 33, pp. 897-906.

[4] Global asthma report 2018. Electronic resource: Available from:

http://www.globalasthmareport.org/Global\%20Asthma\%20Re port $\% 202018$.pdf.

[5] Bel E. H. Clinical phenotypes of asthma. Curr. Opin. Med., 2004; 10, pp. 44-50.

[6] Sara Agnafors, Anna Norman Kjellström, Jarl Torgerson and Marie Rusner, Somatic comorbidity in children and adolescents with psychiatric disorders, European Child \& Adolescent Psychiatry, 2019. 10.1007/s00787-019-01313-9.

[7] Thomas Mike, Bruton Anne, Moffatt Mandy, Cleland Jennifer. Asthma and psuchological dysfunction. - Primary Care Respiratory Journal, 2011, Vol. 20, № 3, pp. 250-256.

[8] Soo Yeon Kim, Sun Ha Choi, Jong Deok Kim, In Suk Sol, Min Jung Kim, Yoon Hee Kim, Young-Chul Jung, Myung Hyun Sohn and Kyung Won Kim, Korean Youth with Comorbid Allergic Disease and Obesity Show Heightened Psychological Distress, The Journal of Pediatrics, 2018. 10.1016/j.jpeds.2018.10.037.

[9] Retyunsky K. Yu., Petrenko T. S., Guryeva A. K. Mental disorders in bronchial asthma: scientific substantiation of the mechanisms of the initiation (science review). Psychiatry and Psychopharmacotherapy, 2018, Vol. 20, № 5, pp. 44-51.

[10] Farzin Irani, Cognitive Functioning in Asthma: Central Nervous System and Other Influences, Handbook of Medical Neuropsychology, 2019, Vol. 10, pp. 187-200.

[11] Gennaro Liccardi, Luigino Calzetta, Antonello Salzillo, Amedeo Piccolo, Gerardo Apicella and Paola Rogliani, Anxiety and asthma in youth. Is a stress - induced increased cholinergic tone the possible link?, Pediatric Pulmonology, 2017. Vol. 53, № 2, pp. 128-129.

[12] Muramatsu Yoshiyuki, Muramatsu Kumiko, Mashima Ichiro, Gejyo Fumitaki. Bronchial Asthma: Psychosomatic aspect. The Journal of the Japan Medical Association, 2001. Vol 126. 3. 375-377.

[13] The Brussels Declaration: the need for change in asthma management / S. Holgate, H. Bisgaard, L. Bjermer, T. Hashtela et al. Europian Respiratory Journal, 2008, Vol. 32, pp. 1433-1442. 
[14] Gary James Connett, Lucy Anne Connett and Mike Thomas, Determining the reasons for poorly controlled asthma in an adolescent. BMJ, 2019. 10.1136/bmj.175, p. 175.

[15] Ashley H. Clawson, Nicole Ruppe, Cara Nwankwo, Alexandra Blair, Marissa Baudino and Nighat Mehdi, Cognitive Behavioral Therapy for Youth with Asthma: Anxiety as an Example. Handbook of Cognitive Behavioral Therapy for Pediatric Medical Conditions, 2019. 10.1007/978-3-030-21683-2(22), pp. 345-367.

[16] Hirokazu Kurahashi, Akihisa Okumura. Teruko Koide. Yoshihiro Ando, Hidehiko Hirata. Miyuki Magota, Kazuyoshi Watabane. Posterior reversible encephalopathy syndrome in a child with bronchial asthma. Electronic resource: Available from:

https://www.sciencedirect.com/science/article/abs/pii/S03877 $6040600057 X$.

[17] Massimiliano Buoli and Alice Caldiroli, When is pharmacotherapy required for children suffering from Generalized Anxiety Disorder? Expert Opinion on Pharmacotherapy, 2019, Vol. 20, № 9, pp. 1053-1056.

[18] Kew K. M., Nashed M., Dulay V., Yorke J. (2016) Cognitive behavioral therapy (CBT) for adults and adolescents with asthma. Cochrane Database of Systematic Reviews, issue 9: CD011818. - $\quad$ Electronic resource: http:www.ncbi.nlm.nih.gov/pubmed/27649894.

[19] Asthma Control Test, Quality Metric Incorporated. Electronic resource: Available from: https://www.memphischildrens.org/Asthma_Control-12-andolder.pdf.

[20] Miller M. R., Hankinson J., Brusasco V., Burgos F., Casaburi R. Et al. Standardisation of spirometry. - Eur. Respir. Journ., 2005, Vol. 26? pp. 319-368.

[21] Schmieschek, H. Fragenbogen zur Ermittlung akzentuirter Personlichkeiten Psychiatr., Neurol und med. Psychol., 1970, Vol. 10, pp. 167-179.

[22] Rean A. A. Prakticheskaya psikhodiagnostika lichnosti: [ucheb. posobie] - SPb.: S.-Peterb. universitet, 2001, 224 pp.

[23] Afobazole [Fabomotizole], Instruction for use, contraindications, composition. - Electronic resource: https://nootropicspot.com/product/afobazole-fabomotizole/.

[24] Thiocetam (Piracetam/Thiotriazoline) Instruction for use, contraindications, composition. - Electronic resource: https://compendium.com.ua/dec/260868/

[25] Gromova O. A., Torshin I. Yu., Pronin A. V., Volkov A. Yu. Synergistic neurotrophic effects of piracetam and thiotriazoline. Neurologia, neiropsikhiatria, psikhosomatika=Neurology, neuropsychiatry, psychosomatics, 2016, Vol. 8, № 16 pp. 86-89.

[26] Medical Outcomes Study Questionnaire Short Form 36 Health Survey (SF-36). -Electronic resource: Available from:
https://www.brandeis.edu/roybal/docs/SF36_website_PDF.pdf.

[27] Buhl Achim, Zofel Peter. SPSS Vertion 10: Eifuhrung in die modern Datenanalyse unter Windows: 7., uberarbeitete und erweiterte Auflage. - Copyright 2000 by Pearson Education Deutschland GmbH. All rights reserved. - SPb.: OOO "DiaSoftYuP", 2005. - 608 pp.

\section{Biography}

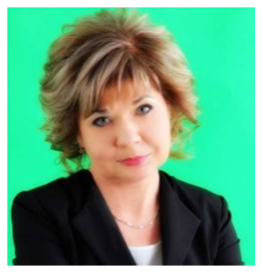

Tetyana Konstantynovych $(\mathrm{MScD})$ is a professor of the Propedeutic Department to internal medicine of National Pirogov Memorial Medical University, Vinnytsya, Ukraine. She works at the areas of Pulmonology, Asthmatology, Cardiology and comorbid pathology, Psychology. Her works have been published in academic journals of Ukraine, Georgia and India, the Journals of the European Respiratory Society.

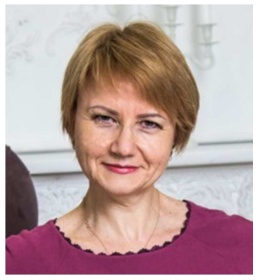

Anna Demchuk (MScD) is a professor of Propedeutic Department to internal medicine of National Pirogov Memorial Medical University, Vinnytsya, Ukraine. She works at the areas of Pulmonology, bacteriology and antibiotics Pharmacology, Cardiology, comorbid pathology. Her works have been published in academic journals of Ukraine, Georgia and India, the journals of the European Respiratory Society.

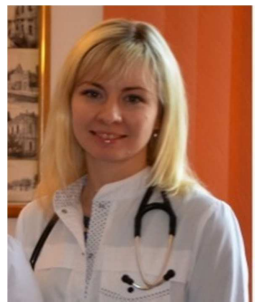

Alina Dovgan $(\mathrm{PhD})$ is an assistant of the Propedeutic Department to internal medicine of National Pirogov Memorial Medical University, Vinnytsya, Ukraine. She works at the areas of Pulmonology, Cardiology, Psychology. Her works have been published in academic journals of Ukraine and the journals of the European Respiratory Society.

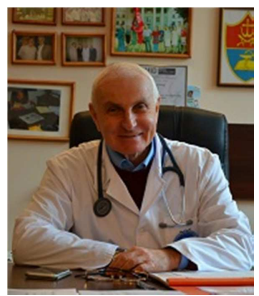

Yuriy Mostovoy (MScD, professor) is a head of the Propedeutic Department to internal medicine of National Pirogov Memorial Medical University, Vinnytsya, Ukraine. He works at the areas of Pulmonology, Asthmatology, Cardiology, Endocrinology, Intensive Care and comorbid pathology. His works have been published in academic journals of Ukraine, the USA, the United Kingdom, Georgia and India, the journals of the European Respiratory Society. 